JOURNAL OF

FUNCTION SPACES AND APPLICATIONS

Volume 9, Number 1 (2011), 1-16
(C) 2011, Scientific Horizon

http://www.jfsa.net

\title{
Weighted holomorphic Besov spaces on the polydisk
}

\section{Anahit V. Harutyunyan ${ }^{1}$ and Wolfgang Lusky}

(Communicated by Jurgen Appell)

2000 Mathematics Subject Classification. 32C37, 47B38, 47B06, 46T25, $46 \mathrm{E} 15$.

Keywords and phrases. Weighted Besov spaces; polydisk; projection.

Abstract. This work is an introduction of weighted Besov spaces of holomorphic functions on the polydisk. Let $U^{n}$ be the unit polydisk in $C^{n}$ and $S$ be the space of functions of regular variation. Let $1 \leq p<\infty, \omega=\left(\omega_{1}, \ldots, \omega_{n}\right)$, $\omega_{j} \in S(1 \leq j \leq n)$ and $f \in H\left(U^{n}\right)$. The function $f$ is said to be an element of the holomorphic Besov space $B_{p}(\omega)$ if

$$
\|f\|_{B_{p}(\omega)}^{p}=\int_{U^{n}}|D f(z)|^{p} \prod_{j=1}^{n} \frac{\omega_{j}\left(1-\left|z_{j}\right|\right)}{\left(1-\left|z_{j}\right|^{2}\right)^{2-p}} d m_{2 n}(z)<+\infty,
$$

where $d m_{2 n}(z)$ is the $2 n$-dimensional Lebesgue measure on $U^{n}$ and $D$ stands for a special fractional derivative of $f$ defined in the paper. For example, if $n=1$ then $D f$ is the derivative of the function $z f(z)$.

We describe the holomorphic Besov space in terms of $L_{p}(\omega)$ space. Moreover projection theorems and theorems of the existence of a right inverse are proved.

\section{Introduction}

In the present article holomorphic weighted Besov spaces on the polydisk are introduced. Numerous authors have contributed to holomorphic Besov spaces in the unit disk in $C$ and in the unit ball in $C^{n}$, see K. Zhu [15], K. Stroethoff [13], Arazy-Fisher-Peetre [2], O. Blasco [3], A. Karapetyants [9].

\footnotetext{
${ }^{1}$ Supported by DFG 436 ARM 17/2/06
} 
The investigation of holomorphic Besov spaces on the polydisk is of special interest. The polydisk is a product of $n$ disks. So one would expect that the results of the one-dimensional case are also valid in the $n$-dimensional case. But it turns out that this is not true. The polydisk case is different from the one-dimensional case and from the case of the $n$ - dimensional ball. For example, let us consider the classical theorem of Privalov: if $f \in \operatorname{Lip} \alpha$, then $K f \in \operatorname{Lip} \alpha$, where $K f$ is a Cauchy type integral. It is known that the analogue of this theorem for multidimensional Lipschitz classes is not true ([14]), even though the analogue of this theorem for a sphere is valid ([10]). In many cases, especially, when the class is defined by means of derivatives, the generalisation of function spaces in the polydisk is different from that on the unit ball. Our aim is to generalize the notion of Besov space in such way that all the known classical results of the one-variable case remain true here (for instance the description of continuous linear functionals, the projection theorems, etc.). Moreover, our generalisation will give results which are new also for functions of one variable.

In section 2, we give definitions and prove auxiliary lemmas. Note that in contrast to the one-dimensional non-weighted case, the use of weights allows us to define the Besov space as well for $p=1$.

In section 3, we describe the holomorphic Besov space in terms of $L_{p}(\omega)$ norms.

In section 4, projection theorems and theorems of the existence of a right inverse are proved.

Based on the results of this paper we shall characterize the duals of our Besov spaces in [7].

\section{Definitions and auxiliary constructions}

Denote by

$$
U^{n}=\left\{z=\left(z_{1}, \ldots, z_{n}\right) \in C^{n},\left|z_{j}\right|<1,1 \leq j \leq n\right\}
$$

the unit polydisk in the $n$-dimensional complex plane $C^{n}$, and by

$$
T^{n}=\left\{z=\left(z_{1}, \ldots, z_{n}\right) \in C^{n},\left|z_{i}\right|=1,1 \leq i \leq n\right\}
$$

its torus.

Definition 1. Let $S$ be the class of all non-negative measurable functions $\omega$ on $(0,1)$ with

$$
\omega(x)=\exp \left\{\eta(x)+\int_{x}^{1} \frac{\varepsilon(u)}{u} d u\right\}, x \in(0,1)
$$


where $\varepsilon$ and $\eta$ are measurable, bounded function on $(0,1)$. We assume that there are constants $\beta_{\omega}<0$ and $\alpha_{\omega}$ with

$$
-\alpha_{\omega} \leq \varepsilon(u) \leq \beta_{\omega} \quad \text { for all } \quad u \in(0,1)
$$

The functions in $S$ are called functions of regular variation (see [11]).

Note that $\omega \in S$ always satisfies

$$
e^{\inf _{t} \eta(t)} x^{\alpha_{\omega}} \leq \omega(x) \leq e^{\sup _{t} \eta(t)} x^{-\beta_{\omega}}, \quad x \in(0,1)
$$

Let $\omega(t)=\left(\omega_{1}(t), \ldots, \omega_{n}(t)\right)$. Throughout the paperwe assume that $\omega_{j} \in S, \beta_{\omega_{j}}<-1(1 \leq j \leq n)$ and $\omega(1-|z|)=\prod_{j=1}^{n} \omega_{j}\left(1-\left|z_{j}\right|\right)$. Further, for $\alpha=\left(\alpha_{1}, \ldots, \alpha_{n}\right), \zeta=\left(\zeta_{1}, \ldots, \zeta_{n}\right), z=\left(z_{1}, \ldots, z_{n}\right)$ we use the following multi-index notations:

$\alpha+r=\left(\alpha_{1}+r, \ldots, \alpha_{n}+r\right)$, if $r$ is a real number, and

$(1-|z|)^{\alpha}=\prod_{j=1}^{n}\left(1-\left|z_{j}\right|\right)^{\alpha_{j}},(1-\bar{\zeta} z)^{\alpha}=\prod_{j=1}^{n}\left(1-\bar{\zeta}_{j} z_{j}\right)^{\alpha_{j}}, z^{k}=$ $z_{1}^{k_{1}} \ldots z_{n}^{k_{n}}$.

Besides, for any function $f$ and $g$ the notation $f \preceq g(f \succeq g)$ will mean that $|f(z)| \leq C|g(z)|(|g(z)| \leq C|f(z)|)$ and the notation $f \asymp g$ will mean that $C_{1}|f(z)| \leq|g(z)| \leq C_{2}|f(z)|$ for some positive constants $C, C_{1}, C_{2}$ independent of $z$.

Note that it is not difficult to show that $\omega(1-|z|) \asymp \omega\left(1-|z|^{2}\right)$.

Let $H\left(U^{n}\right)$ be the set of holomorphic functions on $U^{n}$.

Definition 2. Let $p \geq 1 \alpha=\left(\alpha_{1}, \ldots, \alpha_{n}\right), \alpha_{j}>-1(1 \leq j \leq n)$. We denote by $A^{p}(\alpha)$, the set of all $f \in H\left(U^{n}\right)$, for which

$$
\|f\|_{A^{p}(\alpha)}^{p}=\int_{U^{n}}|f(z)|^{p}(1-|z|)^{\alpha} d m_{2 n}(z)<+\infty
$$

where $d m_{2 n}(z)$ is the $2 n$ - dimensional Lebesgue measure on $U^{n}$.

The integral representation formula for the class $A^{p}(\alpha)$ is a trivial consequence of the well known one-dimensional case:

$$
f(z)=\frac{\prod_{j=1}^{n}\left(\alpha_{j}+1\right)}{\pi^{n}} \int_{U^{n}} \frac{\left(1-|\zeta|^{2}\right)^{\alpha}}{(1-\bar{\zeta} z)^{\alpha+2}} f(\zeta) d m_{2 n}(\zeta)
$$

for details see [4], [12]. Note that the generalization of $A_{\alpha}^{p}$ spaces in terms of $\omega$ weights was used for the first time by F. A. Shamoyan [12] who greatly invested in the theory of weighted classes of functions in the polydisk. 
Definition 3. Let $p \geq 1$. We denote by $L_{p}(\omega)$ the set of all measurable functions on $U^{n}$, for which

$$
\|f\|_{L_{p}(\omega)}^{p}=\int_{U^{n}}|f(z)|^{p} \frac{\omega(1-|z|)}{\left(1-|z|^{2}\right)^{2}} d m_{2 n}(z)<+\infty .
$$

Notice that $L_{p}(\omega)$ is the $L_{p}$-space with respect to the measure $\omega(1-|z|)\left(1-|z|^{2}\right)^{-2} d m_{2 n}(z)$. In view on our conditions on $\omega\left(\omega_{j} \in S\right)$ this measure is bounded.

The following definition gives the notion of the fractional differential.

Definition 4. For a holomorphic function $f(z)=\sum_{(k)=(0)}^{(\infty)} a_{k} z^{k}, \quad z \in$ $U^{n}$, and for $\beta=\left(\beta_{1}, \ldots, \beta_{n}\right), \beta_{j}>-1(1 \leq j \leq n)$

(i) we define the fractional differential $D^{\beta}$ as follows

$$
D^{\beta} f(z)=\sum_{(k)=(0)}^{(\infty)} \prod_{j=1}^{n} \frac{\Gamma\left(\beta_{j}+1+k_{j}\right)}{\Gamma\left(\beta_{j}+1\right) \Gamma\left(k_{j}+1\right)} a_{k} z^{k}, \quad k=\left(k_{1}, \ldots, k_{n}\right), \quad z \in U^{n},
$$

where $\Gamma($.$) is the Gamma function and \sum_{(k)=(0)}^{(\infty)}=\sum_{k_{1}=0}^{\infty} \ldots \sum_{k_{n}=0}^{\infty}$.

(ii) Let $D^{-\beta}$ be the inverse operator: $D^{-\beta} D^{\beta} f(z)=f(z), z \in U^{n}$.

In particular, if the $\beta_{j}$ are positive integers, then

$$
D^{\beta} f(z)=\left(\prod_{j=1}^{n} \frac{1}{\Gamma\left(\beta_{j}+1\right)}\right) \frac{\partial^{\beta_{1}+\ldots+\beta_{n}}\left(f(z) z^{\beta}\right)}{\partial z_{1}^{\beta_{1}} \ldots \partial z_{n}^{\beta_{n}}}
$$

We have

$$
D^{-\beta} f(z)=\sum_{(k)=(0)}^{(\infty)} \prod_{j=1}^{n} \frac{\Gamma\left(\beta_{j}+1\right) \Gamma\left(k_{j}+1\right)}{\Gamma\left(\beta_{j}+1+k_{j}\right)} a_{k} z^{k} .
$$

If $\beta=(1, \ldots, 1)$ then we put $D^{\beta} f(z) \equiv D f(z)$.

Hence

$$
D f\left(z_{1}, \ldots, z_{n}\right)=\frac{\partial^{n}\left(f\left(z_{1}, \ldots, z_{n}\right) z_{1} \cdots z_{n}\right)}{\partial z_{1} \ldots \partial z_{n}} .
$$

Now we define holomorphic Besov spaces on the polydisk.

Definition 5. Let $1 \leq p<\infty$ and $f \in H\left(U^{n}\right)$. The function $f$ is said to be in $B_{p}(\omega)$ if

$$
\|f\|_{B_{p}(\omega)}=\left(\int_{U^{n}}|D f(z)|^{p} \frac{\omega(1-|z|)}{\left(1-|z|^{2}\right)^{2-p}} d m_{2 n}(z)\right)^{1 / p}<+\infty
$$


Notice that, in view of our definition of $D f,\|\cdot\|_{B_{p}(\omega)}$ is indeed a norm. (We do not have to add $|f(0)|$ ). This follows from the fact that here $D f=0$ implies $f=0$ for holomorphic $f$.

Definition 5 is closely related to the classical Besov space where $p>$ $1, n=1$, and $\omega(t)=1$ (see [15]) and to the classical Dirichlet space where $p=2([1])$. Indeed, recall that the norm of the classical Besov space is defined by

$$
\||f|\|_{p}=\left(\int_{\mathbb{D}}\left(1-|z|^{2}\right)^{p}\left|f^{\prime}(z)\right|^{p} d \lambda(z)\right)^{1 / p} \quad \text { where } \quad d \lambda(z)=\frac{d m_{2}(z)}{\left(1-|z|^{2}\right)^{2}}
$$

and $p>1$. Hence, according to Definition $5,\left|\|z f \mid\|_{p}=\|f\|_{B_{p}(\omega)}\right.$ where $\omega(t)=1$. However, in our paper we require $\omega_{j} \in S$ and $\beta_{\omega}<-1$. This means in particular that $\omega(1-|z|)$ tends to 0 as $|z|$ tends to 1 . We clearly have that $B_{p}(\omega)$ is a subset of $B_{1}(\omega)$ for any $p>1$.

As in the one dimensional case, $B_{p}(\omega)$ is a Banach space with respect to the norm (3).

To prove the main results we need the following auxiliary lemma:

Lemma 1. Let $m=\left(m_{1}, \ldots, m_{n}\right)$ and $\beta=\left(\beta_{1}, \ldots, \beta_{n}\right) \in \mathbb{N}^{n}$. Then the following statements are true

1. If $f \in H\left(U^{n}\right)$ and $\left(1-|z|^{2}\right)^{\beta} D^{\beta} f \in L_{1}(\omega)$, then

$$
|D f(z)| \preceq \int_{U^{n}} \frac{\left(1-|\zeta|^{2}\right)^{m}}{|1-\bar{\zeta} z|^{m+3-\beta}}\left|D^{\beta} f(\zeta)\right| d m_{2 n}(\zeta),
$$

where $m_{j} \geq \beta_{j}+\alpha_{\omega_{j}}-2(1 \leq j \leq n)$.

2. If $f \in B_{1}(\omega)$, then

$$
\left|D^{\beta} f(z)\right| \preceq \int_{U^{n}} \frac{\left(1-|\zeta|^{2}\right)^{m}}{|1-\bar{\zeta} z|^{m+1+\beta}}|D f(\zeta)| d m_{2 n}(\zeta)
$$

where $m_{j} \geq \alpha_{\omega_{j}}-2(1 \leq j \leq n)$.

Proof. 1. Let $f \in H\left(U^{n}\right), f(z)=\sum_{(k)=(0)}^{(\infty)} a_{k} z^{k}$ such that the function $\left(1-|z|^{2}\right)^{\beta} D^{\beta} f(z)$ belongs to $L_{p}(\omega)$. Then $D^{\beta} f \in A^{1}(m)$ for $m_{j} \geq \beta_{j}+\alpha_{\omega_{j}}-2(1 \leq j \leq n)$ and has the representation (1) for $\alpha_{j}=m_{j}(1 \leq j \leq n)$. By definition of $D^{\beta}$ one can see that $D D^{-\beta} D^{\beta} f(z)=$ $D f(z)$.

Then, using (1) for $D^{\beta} f$ and applying $D D^{-\beta}$ on both sides, we obtain

$D f(z)=\frac{\prod_{j=1}^{n}\left(m_{j}+1\right)}{\pi^{n}} \int_{U^{n}}\left(1-|\zeta|^{2}\right)^{m} D D^{-\beta} \frac{1}{(1-\bar{\zeta} z)^{m+2}} D^{\beta} f(\zeta) d m_{2 n}(\zeta)$. 
A calculation shows that

$$
D^{-\beta} \frac{1}{(1-\bar{\zeta} z)^{m+2}}=\int_{0}^{1} \frac{(1-r)^{\beta-1}}{(1-r \bar{\zeta} z)^{m+2}} d r
$$

By partial integration we get

$$
D^{-\beta} \frac{1}{(1-\bar{\zeta} z)^{m+2}}=\frac{P(\bar{\zeta} z)}{(1-r \bar{\zeta} z)^{m+2-\beta}}
$$

where $P(\bar{\zeta} z)$ is some Polynom of order $m+1-\beta$. Then using (2) we get

$$
D\left(D^{-\beta}\right) \frac{1}{(1-\bar{\zeta} z)^{m+2}} \preceq \frac{1}{(1-\bar{\zeta} z)^{m+3-\beta}},
$$

which proves (4).

For the proof of (5) use the fact that $D f \in A^{1}(m)$ if $m_{j} \geq \alpha_{\omega}-2$ and $1 \leq j \leq n$. Apply (1) to $D f$ and then apply $D^{\beta} D^{-1}$ on both sides. Similarly to the proof of the first part of this lemma we obtain (5).

The proofs of the main theorems are also based on the following lemma.

Lemma 2. Let $n=1$.

a) Assume $a+1-\beta_{\omega}>0, b>1$ and $b-a-2>\alpha_{\omega}$. Then

$$
\int_{U} \frac{\left(1-|\zeta|^{2}\right)^{a} \omega\left(1-|\zeta|^{2}\right)}{|1-z \bar{\zeta}|^{b}} d m_{2}(\zeta) \preceq \frac{\omega\left(1-|z|^{2}\right)}{\left(1-|z|^{2}\right)^{b-a-2}}
$$

b) If $b>1$ and $b-a>2$ then

$$
\int_{U} \frac{\left(1-|\zeta|^{2}\right)^{a}}{|1-z \bar{\zeta}|^{b}} d m_{2}(\zeta) \preceq \frac{1}{\left(1-|z|^{2}\right)^{b-a-2}}
$$

For the proof of a) see [6], Lemma 1.6, for b) see [15], Theorem 1.7.

\section{Relations of $B_{p}(\omega)$ to weighted Lebesgue spaces}

As the first property, we prove that $B_{p}(\omega)$ is a subspace of $L_{p}(\omega)$ for any $p$.

Theorem 1. $B_{p}(\omega) \subset L_{p}(\omega)$ and $\|f\|_{L_{p}(\omega)} \preceq\|f\|_{B_{p}(\omega)}$ for $1 \leq p<\infty$.

Proof. Let $f \in B_{p}(\omega)$. At first assume $p>1$. Since $B_{p}(\omega)$ is a subspace of $B_{1}(\omega)$ we have $f \in B_{1}(\omega)$. Then using the inequality (5) with $\beta=0$ 
and $m+\delta$ instead of $m$ we get

$$
|f(z)|^{p} \preceq\left(\int_{U^{n}} \frac{\left(1-|\zeta|^{2}\right)^{m-1}}{|1-\bar{\zeta} z|^{m+1+\delta}}|D f(\zeta)|\left(1-|\zeta|^{2}\right)^{1+\delta} d m_{2 n}(\zeta)\right)^{p},
$$

where $\delta>0$ and $\delta p$ is sufficiently small. The Hölder inequality with $1 / q+1 / p=1$ yields

$$
\begin{aligned}
|f(z)|^{p} \preceq & \left(\int_{U^{n}} \frac{(1-|\zeta|)^{m-1}}{|1-\bar{\zeta} z|^{m+1+\delta q}} d m_{2 n}(\zeta)\right)^{p / q} \times \\
& \times\left(\int_{U^{n}} \frac{\left(1-|\zeta|^{2}\right)^{m-1+\delta p}}{|1-\bar{\zeta} z|^{m+1}}|D f(\zeta)|^{p}\left(1-|\zeta|^{2}\right)^{p} d m_{2 n}(\zeta)\right) \\
\preceq & \frac{1}{\left(1-|z|^{2}\right)^{\delta p}} \int_{U^{n}} \frac{\left(1-|\zeta|^{2}\right)^{m-1+\delta p}}{|1-\bar{\zeta} z|^{m+1}}|D f(\zeta)|^{p}\left(1-|\zeta|^{2}\right)^{p} d m_{2 n}(\zeta)
\end{aligned}
$$

Here we used Lemma 2 b). With Lemma 2 we also get

$$
\begin{aligned}
\int_{U^{n}}|f(z)|^{p} & \frac{\omega(1-|z|)}{\left(1-|z|^{2}\right)^{2}} d m_{2 n}(z) \\
\preceq & \int_{U^{n}}\left(1-|\zeta|^{2}\right)^{m-1+\delta p+p}|D f(\zeta)|^{p} \times \\
& \quad \times \int_{U^{n}} \frac{\omega(1-|z|)\left(1-|z|^{2}\right)^{-\delta p-2}}{|1-\bar{\zeta} z|^{m+1}} d m_{2 n}(z) d m_{2 n}(\zeta) \\
\preceq & \int_{U^{n}}|D f(\zeta)|^{p} \frac{\omega(1-|\zeta|)}{\left(1-|\zeta|^{2}\right)^{2-p}} d m_{2 n}(\zeta)=\|f\|_{B_{p}(\omega)}<\infty .
\end{aligned}
$$

If $p=1$ then (5) with $\beta=0$ yields

$$
\begin{aligned}
\int_{U^{n}} & |f(z)| \frac{\omega(1-|z|)}{\left(1-|z|^{2}\right)^{2}} d m_{2 n}(z) \\
& \preceq \int_{U^{n}}\left(1-|\zeta|^{2}\right)^{m}|D f(\zeta)| \int_{U^{n}} \frac{\omega(1-|z|)\left(1-|z|^{2}\right)^{-2}}{|1-\bar{\zeta} z|^{m+1}} d m_{2 n}(z) d m_{2 n}(\zeta) \\
& \preceq \int_{U^{n}}|D f(\zeta)| \frac{\omega(1-|\zeta|)}{\left(1-|\zeta|^{2}\right)} d m_{2 n}(\zeta)=\|f\|_{B_{1}(\omega)}<\infty
\end{aligned}
$$

Here we used Lemma 2 again.

Theorem 2. Let $f \in H\left(U^{n}\right), \beta_{j}>1(1 \leq j \leq n)$ and $1 \leq p<\infty$. Then $f \in B_{p}(\omega)$ if and only if $g \in L_{p}(\omega)$, where $g(z)=\left(1-|z|^{2}\right)^{\beta} D^{\beta} f(z), z \in$ $U^{n}$. Moreover, $\|f\|_{B_{p}(\omega)} \asymp\|g\|_{L^{p}(\omega)}$. 
Proof. Let $f \in B_{p}(\omega)$. Since then $f \in B_{1}(\omega)$ we can apply (5). We get

$$
\left|D^{\beta} f(z)\right| \preceq \int_{U^{n}} \frac{\left(1-|\zeta|^{2}\right)^{m}|D f(\zeta)|}{|1-\bar{\zeta} z|^{m+1+\beta}} d m_{2 n}(\zeta)
$$

We consider the cases $p>1$ and $p=1$ separately.

Let $p>1$. Using Hölder's inequality we get from (6) with Lemma 2

$$
\left|D^{\beta} f(z)\right|^{p} \preceq \frac{1}{\left(1-|z|^{2}\right)^{(\beta-1) p / q}} \int_{U^{n}} \frac{\left(1-|\zeta|^{2}\right)^{m}|D f(\zeta)|^{p}}{|1-\bar{\zeta} z|^{m+1+\beta}} d m_{2 n}(\zeta) .
$$

Therefore

$$
\begin{aligned}
\|g\|_{L_{p}(\omega)}= & \int_{U^{n}}|g(z)|^{p} \frac{\omega(1-|z|)}{\left(1-|z|^{2}\right)^{2}} d m_{2 n}(z) \\
\preceq & \int_{U^{n}}\left(1-|\zeta|^{2}\right)^{m}|D f(\zeta)|^{p} \times \\
& \times \int_{U^{n}} \frac{\left(1-|z|^{2}\right)^{\beta p-(\beta-1) p / q-2}}{|1-\bar{\zeta} z|^{m+1+\beta}} \omega(1-|z|) d m_{2 n}(z) d m_{2 n}(\zeta) .
\end{aligned}
$$

Using Lemma 2 we have

$$
\|g\|_{L_{p}(\omega)} \preceq \int_{U^{n}}\left(1-|\zeta|^{2}\right)^{p-2}|D f(\zeta)|^{p} \omega(1-|\zeta|) d m_{2 n}(\zeta)=\|f\|_{B_{p}(\omega)}<\infty
$$

Let $p=1$. Then by Fubini theorem and (6) we get

$$
\begin{aligned}
\|g\|_{L_{1}(\omega)}= & \int_{U^{n}}|g(z)| \frac{\omega(1-|z|)}{\left(1-|z|^{2}\right)} d m_{2 n}(z) \\
\preceq & \int_{U^{n}}\left(1-|\zeta|^{2}\right)^{m}|D f(\zeta)| \times \\
& \times \int_{U^{n}} \frac{\left(1-|z|^{2}\right)^{\beta-2} \omega(1-|z|)}{|1-\bar{\zeta} z|^{m+1+\beta}} d m_{2 n}(z) d m_{2 n}(\zeta) \\
\preceq & \int_{U^{n}}\left(1-|\zeta|^{2}\right)^{m}|D f(\zeta)| \frac{\left(1-|\zeta|^{2}\right)^{\beta-2} \omega(1-|\zeta|)}{\left(1-|\zeta|^{2}\right)^{m-1+\beta}} d m_{2 n}(\zeta) \\
= & \int_{U^{n}}|D f(\zeta)| \frac{\omega(1-|\zeta|)}{\left(1-|\zeta|^{2}\right)} d m_{2 n}(\zeta)=\|f\|_{B_{1}(\omega)}<\infty .
\end{aligned}
$$

In the last inequality we have used Lemma 2 again. Summing up, we have proved, that $g \in L_{p}(\omega)$ and

$$
\|g\|_{L^{p}(\omega)} \leq C_{2}(\omega, p)\|f\|_{B_{p}(\omega)} .
$$


Conversely, let $f \in H\left(U^{n}\right)$ and $g \in L^{p}(\omega)$. Then using (4) and Lemma 2 , in the case $p>1$, we have

$$
|D f(z)|^{p} \preceq \frac{1}{\left(1-|z|^{2}\right)^{p / q}} \int_{U^{n}} \frac{\left(1-|\zeta|^{2}\right)^{m-\beta}}{|1-\bar{\zeta} z|^{m-\beta+3}}|g(\zeta)|^{p} d m_{2 n}(\zeta) .
$$

Then

$$
\begin{aligned}
& \int_{U^{n}}\left(1-|z|^{2}\right)^{p-2-p / q} \omega(1-|z|) \int_{U^{n}} \frac{\left(1-|\zeta|^{2}\right)^{m-\beta}}{|1-\bar{\zeta} z|^{m-\beta+3}}|g(\zeta)|^{p} d m_{2 n}(\zeta) d m_{2 n}(z) \\
& \quad=\int_{U^{n}}\left(1-|\zeta|^{2}\right)^{m-\beta}|g(\zeta)|^{p} \int_{U^{n}} \frac{\left(1-|z|^{2}\right)^{p-2-p / q}}{|1-\bar{\zeta} z|^{m-\beta+3}} \omega(1-|z|) d m_{2 n}(z) d m_{2 n}(\zeta) \\
& \quad=\int_{U^{n}}|g(\zeta)|^{p} \frac{\omega(1-|\zeta|)}{\left(1-|\zeta|^{2}\right)^{2}} d m_{2 n}(\zeta)=\|g\|_{L^{p}(\omega)}<\infty
\end{aligned}
$$

This proves $\|f\|_{B_{p}(\omega)} \preceq\|g\|_{L^{p}(\omega)}$.

In the case $p=1$ we have, using Lemma 2 ,

$$
\begin{aligned}
& \int_{U^{n}}\left(1-|\zeta|^{2}\right)^{m}\left|D^{\beta}(\zeta)\right| \int_{U^{n}} \frac{\left(1-|z|^{2}\right)^{-1}}{|1-\bar{\zeta} z|^{m-\beta+3}} \omega(1-|z|) d m_{2 n}(z) d m_{2 n}(\zeta) \\
& \quad \preceq \int_{U^{n}}\left(1-|\zeta|^{2}\right)^{m}\left|D^{\beta}(\zeta)\right|\left(1-|\zeta|^{2}\right)^{-1-m-1+\beta} \omega(1-|\zeta|) d m_{2 n}(\zeta) \\
& \quad=\int_{U^{n}}|g(\zeta)| \frac{\omega(1-|\zeta|)}{\left(1-|\zeta|^{2}\right)^{2}} d m_{2 n}(\zeta)=\|g\|_{L_{1}(\omega)}<\infty
\end{aligned}
$$

and therefore $\|f\|_{B_{1}(\omega)} \preceq\|g\|_{L_{1}(\omega)}$. So we have proved that $f \in B_{p}(\omega)$ and

$$
\|f\|_{B_{p}(\omega)} \leq C_{1}(\omega, p)\|g\|_{L^{p}(\omega)}
$$

From (7) and (8) we get $\|f\|_{B_{p}(\omega)} \asymp\|g\|_{L^{p}(\omega)}$.

Let $\widetilde{B}_{1}\left(\omega_{i_{1}}, \ldots, \omega_{i_{k}}\right)$ be the class of $f \in H\left(U^{n}\right)$ with $\|f\|_{\widetilde{B}_{1}\left(\omega_{i_{1}} \ldots \omega_{i_{k}}\right)}<$ $\infty$, where

$$
\|f\|_{\widetilde{B}_{1}\left(\omega_{i_{1}} \ldots \omega_{i_{k}}\right)}=\int_{U^{n}} \prod_{j=1}^{k}\left(1-\left|z_{i_{j}}\right|^{2}\right)\left|\frac{\partial^{k} f(z)}{\partial z_{i_{1}} \ldots \partial z_{i_{k}}}\right| \frac{\omega(1-|z|)}{\left(1-|z|^{2}\right)^{2}} d m_{2 n}(z) .
$$

$\widetilde{B}_{1}\left(\omega_{i_{1}} \ldots \omega_{i_{k}}\right)$ will be called holomorphic Besov space with respect to $\left(i_{1}, \ldots, i_{k}\right)$. The next theorem shows how the two Besov spaces $B_{1}$ and $\tilde{B}_{1}$ are related. 
Theorem 3. $f \in B_{1}(\omega)$ if and only if $f \in \widetilde{B}_{1}\left(\omega_{i_{1}}, \ldots, \omega_{i_{k}}\right)$ for all $1 \leq i_{j} \leq n, 1 \leq j \leq k, 1 \leq k \leq n$, and $f \in L_{1}(\omega)$.

Proof. Let $f \in B_{1}(\omega)$. At first assume that $k=n-1,\left(i_{1}, \ldots, i_{k}\right)=$ $(2, \ldots, n)$. Our aim is to show, that the following function belongs to $L_{1}(\omega)$

$$
g(z)=\frac{\partial^{n-1} f(z)}{\partial z_{2} \ldots \partial z_{n}}\left(1-\left|z_{2}^{\prime}\right|^{2}\right), z_{2}^{\prime}=\left(z_{2}, \ldots, z_{n}\right) .
$$

If $m_{j} \geq \alpha_{\omega_{j}}-1, m_{j} \in N(1 \leq j \leq n)$, using the properties of $\omega_{j}$ we obtain $D f \in A^{1}(m)$. Applying (1) to $D f$ and integrating with respect to $z$, we get

$$
f(z)=\frac{1}{\pi^{n}} \int_{U^{n}} \frac{\left(1-|\zeta|^{2}\right)^{m}\left(1-(1-\bar{\zeta} z)^{m+1}\right)}{\bar{\zeta} z(1-\bar{\zeta} z)^{m+1}} D f(\zeta) d m_{2 n}(\zeta),
$$

where the function $\left(1-(1-\bar{\zeta} z)^{m+1}\right) / \bar{\zeta} z$ is bounded and has bounded derivatives on $U^{n}$. Differentiating the last equality we get

$$
\left|\frac{\partial^{n-1} f(z)}{\partial z_{2} \ldots \partial z_{n}}\right| \preceq \int_{U^{n}} \frac{\left(1-|\zeta|^{2}\right)^{m}|D f(\zeta)|}{\left|1-\bar{\zeta}_{1} z_{1}\right|^{m_{1}+1}\left|1-\bar{\zeta}_{2}^{\prime} z_{2}^{\prime}\right|^{m_{2}^{\prime}+2}} d m_{2 n}(\zeta)
$$

where $m_{2}^{\prime}=\left(m_{2}, \ldots, m_{n}\right)$. Then, in view of Lemma 2 ,

$$
\begin{aligned}
\int_{U^{n}} & |g(z)| \frac{\omega(1-|z|)}{\left(1-|z|^{2}\right)^{2}} d m_{2 n}(z) \\
& \preceq \int_{U^{n}}|D f(\zeta)|\left(1-|\zeta|^{2}\right)^{m} \times \\
& \times \int_{U^{n}} \frac{\omega(1-|z|)\left(1-|z|^{2}\right)^{-2}\left(1-\left|z_{2}^{\prime}\right|^{2}\right)}{\left|1-\bar{\zeta}_{1} z_{1}\right|^{m_{1}+1}\left|1-\bar{\zeta}_{2}^{\prime} z_{2}^{\prime}\right|^{m_{2}^{\prime}+2}} d m_{2 n}(z) d m_{2 n}(\zeta) \\
& \preceq \int_{U^{n}}|D f(z)| \frac{\omega(1-|z|)}{\left(1-|z|^{2}\right)} d m_{2 n}(z)=\|f\|_{B_{1}(\omega)}<\infty .
\end{aligned}
$$

Repeating the same argument, we get similarly

$$
\frac{\partial^{k} f(z)}{\partial z_{i_{1}} \ldots \partial z_{i_{k}}} \prod_{j=1}^{k}\left(1-\left|z_{i_{j}}\right|^{2}\right) \in L_{1}(\omega) \quad \text { or } \quad f \in \tilde{B}_{1}\left(\omega_{i_{1}}, \ldots, \omega_{i_{k}}\right),
$$

$1 \leq i_{j} \leq n, 1 \leq j \leq k, 1 \leq k \leq n$, and $f \in L_{1}(\omega)$.

Conversely, if $f \in \widetilde{B}_{1}\left(\omega_{i_{1}}, \ldots, \omega_{i_{k}}\right)$ for all $1 \leq i_{j} \leq n, 1 \leq j \leq k, 1 \leq$ $k \leq n$, and $f \in L_{1}(\omega)$ then in view of

$$
D f(z)=\frac{\partial^{n}(f(z) z)}{\partial z_{1} \ldots \partial z_{n}}=f(z)+\sum \frac{\partial^{k} f(z)}{\partial z_{i_{1}} \ldots \partial z_{i_{k}}} z_{i_{k+1}} \ldots z_{i_{n}}
$$


we get

$$
\begin{aligned}
\int_{U^{n}} \mid & D f(z) \mid \frac{\omega(1-|z|)}{1-|z|^{2}} d m_{2 n}(z) \\
\preceq & \int_{U^{n}}|f(z)| \frac{\omega(1-|z|)}{\left(1-|z|^{2}\right)^{2}} d m_{2 n}(z)+ \\
& +\sum \int_{U^{n}} \prod_{j=1}^{k}\left(1-\left|z_{i_{j}}\right|^{2}\right)\left|\frac{\partial^{k} f(z)}{\partial z_{i_{1}} \ldots \partial z_{i_{k}}}\right| \frac{\omega(1-|z|)}{\left(1-|z|^{2}\right)^{2}} d m_{2 n}(z)<\infty
\end{aligned}
$$

which proves that $\|f\|_{B_{1}(\omega)} \preceq\|f\|_{L_{1}(\omega)}+\sum\|f\|_{\widetilde{B}_{1}\left(\omega_{i_{1}}, \ldots, \omega_{i_{k}}\right)}<\infty$.

\section{Bounded and right inverse operators on $B_{p}(\omega)$}

Let us consider the following linear operator on $L_{p}(\omega)$

$$
P_{\alpha}(g)(z):=\frac{\prod_{j=1}^{n}\left(\alpha_{j}+1\right)}{\pi^{n}} \int_{U^{n}} \frac{\left(1-|\zeta|^{2}\right)^{\alpha}}{(1-\bar{\zeta} z)^{\alpha+2}} g(\zeta) d m_{2 n}(\zeta),
$$

where $\alpha_{j}>-1(1 \leq j \leq n)$. Obviously $P_{\alpha}(g)$ is holomorphic on $U^{n}$. The problem is to characterize the functions $P_{\alpha}(g)$ with $g \in L_{p}(\omega)$. The next theorem shows that $P_{\alpha}$ is bounded on $B_{p}(\omega)$.

Theorem 4. Let $\alpha_{j} p>\alpha_{\omega_{j}}-2(1 \leq j \leq n)$. Then $P_{\alpha}: L_{p}(\omega) \rightarrow B_{p}(\omega)$ is bounded for $1 \leq p<\infty$.

Proof. Let $g \in L_{p}(\omega)$ and $f=P_{\alpha}(g)$. By Theorem 2 it is sufficient to prove that $h \in L_{p}(\omega)$, where $h(z)=(1-|z|)^{\beta} D^{\beta} f(z), z \in U^{n}$ for some $\beta\left(\beta_{j}>1,1 \leq j \leq n\right)$. We have

$$
\left|D^{\beta} f\right| \preceq \int_{U^{n}} \frac{\left(1-|\xi|^{2}\right)^{\alpha}}{|1-\bar{\xi} z|^{\alpha+\beta+2}}|g(\xi)| d m_{2 n}(\xi)
$$

Let $p>1$. Then, with the Hölder inequality $(1 / p+1 / q=1)$ and Lemma 2 ,

$$
\begin{aligned}
\int_{U^{n}} & \left(1-|z|^{2}\right)^{\beta p}\left|D^{\beta} f(z)\right|^{p} \frac{\omega(1-|z|)}{\left(1-|z|^{2}\right)^{2}} d m_{2 n}(z) \\
\preceq & \int_{U^{n}}|g(\zeta)|^{p}\left(1-|\zeta|^{2}\right)^{\alpha p} \times \\
& \times \int_{U^{n}} \frac{\left(1-|z|^{2}\right)^{\beta p-(\alpha+\beta) p / q-2} \omega(1-|z|)}{|1-\bar{\zeta} z|^{\alpha+\beta+2}} d m_{2 n}(z) d m_{2 n}(\zeta)
\end{aligned}
$$




$$
\preceq \int_{U^{n}}|g(\zeta)|^{p}\left(1-|\zeta|^{2}\right)^{\alpha p} \frac{\omega(1-|\zeta|}{\left(1-|\zeta|^{2}\right)^{\alpha p+2}} d m_{2 n}(\zeta) \preceq\|g\|_{L_{p}(\omega)} .
$$

We have used the fact that $\alpha_{j} p>\alpha_{\omega_{j}}-2$ and have taken $\beta_{j}$ so, that $\beta_{j}>\alpha_{j}(p-1)+\beta_{\omega_{j}}+1(1 \leq j \leq n)$.

Let now $p=1$. Then

$$
\begin{aligned}
\int_{U^{n}} & (1-|z|)|D f(z)| \frac{\omega(1-|z|)}{\left(1-|z|^{2}\right)^{2}} d m_{2 n}(z) \\
& \preceq \int_{U^{n}}|g(\zeta)|\left(1-|\zeta|^{2}\right)^{\alpha} \int_{U^{n}} \frac{\left(1-|z|^{2}\right) \omega(1-|z|)}{|1-\bar{\zeta} z|^{\alpha+3}\left(1-|z|^{2}\right)^{2}} d m_{2 n}(z) d m_{2 n}(\zeta) \\
& \preceq \int_{U^{n}}|g(\zeta)| \frac{\omega(1-|\zeta|)}{\left(1-|\zeta|^{2}\right)^{2}} d m_{2 n}(\zeta)=\|g\|_{L_{p}(\omega)}<\infty .
\end{aligned}
$$

Remark 1. The condition $\alpha_{j} p>\alpha_{\omega_{j}}-2(1 \leq j \leq n)$ in Theorem 4 is necessary, too.

Proof of Remark 1. Let $P_{\alpha}$ be bounded from $L_{p}(\omega)$ to $B_{p}(\omega)$ and let

$$
g_{r}(\zeta)=\frac{(1-r)^{k}}{\omega^{1 / p}(1-r)} \frac{1}{|1-r \zeta|^{k}},
$$

$r=\left(r_{1}, \ldots, r_{n}\right), r_{j} \in(1 / 3,1), k_{j}>\alpha_{\omega_{j}}(1 \leq j \leq n)$. Obviously $\left\|g_{r}\right\|_{L_{p}(\omega)} \sim$ const. (in view of Lemma 2 ). We consider the domain

$$
\widetilde{U}_{j}=\left\{z_{j} \in U,\left|\arg z_{j}\right|<1-r_{j} ;\left(3 r_{j}-1\right) / 2<\left|z_{j}\right|<\left(1+r_{j}\right) / 2\right\},
$$

where $(1 \leq j \leq n)$ and $\widetilde{U}^{n}=\widetilde{U}_{1} \times \ldots \times \widetilde{U}_{n}$. Further, let $V^{n}$ be the following polydisk centered at $\left(r_{1}, \ldots, r_{n}\right)$

$$
V^{n}:=\left\{\zeta, \zeta=r+\rho e^{i \theta}, \theta \in[-\pi, \pi], \rho \in(0,(1-r) / 2\} .\right.
$$

Obviously $V^{n} \subset \widetilde{U}^{n}$. Fix $\beta$ such that $\alpha_{j}+\beta_{j}-1>0$. Then, using Theorem 2 and ([8], Theorem 1.9), we get

$$
\left\|\widetilde{P}_{\alpha}\left(g_{r}\right)\right\|_{B_{p}(\omega)} \succeq \int_{U^{n}} \frac{\omega(1-|z|)}{\left(1-|z|^{2}\right)^{2-\beta p}}\left(\int_{\widetilde{U}^{n}} \frac{(1-|\zeta|)^{\alpha}\left|g_{r}(\zeta)\right|}{|1-\bar{\zeta} z|^{\alpha+\beta+2}} d m_{2 n}(\zeta)\right)^{p} d m_{2 n}(z),
$$

where $\widetilde{P}_{\alpha}$ is the corresponding integral from Theorem 1.9 (see [8]). If $\zeta \in \widetilde{U}^{n}$, then it is easy to see that $1-\left|\zeta_{j}\right| \asymp 1-r_{j}(1 \leq j \leq n)$ and $\left|1-r_{j} \zeta_{j}\right| \preceq 1-r_{j}(1 \leq j \leq n)$ using which we get 
$\left\|\widetilde{P}_{\alpha}\left(g_{r}\right)\right\|_{B_{p}(\omega)} \geq \frac{(1-r)^{\alpha p}}{\omega(1-r)} \int_{U^{n}} \frac{\omega(1-|z|)}{\left(1-|z|^{2}\right)^{2-\beta p}}\left(\int_{V^{n}} \frac{d m_{2 n}(\zeta)}{|1-\bar{\zeta} z|^{\alpha+\beta+2}}\right)^{p} d m_{2 n}(z)$

Furthermore, for $a>1$ we have

$$
\int_{-\pi}^{\pi} \frac{d \theta}{\left|1-r \rho e^{-i \theta}\right|^{a}} \sim \frac{1}{(1-r \rho)^{a-1}}, \rho, r \in[0,1) .
$$

Since $\left|r_{j}-\rho_{j}\right| \leq\left|\zeta_{j}\right| \leq\left(\rho_{j}+r_{j}\right)(1 \leq j \leq n)$, for $\zeta \in V^{n}$, we get

$$
\int_{V^{n}} \frac{d m_{2 n}(\zeta)}{|1-\bar{\zeta} z|^{\alpha+\beta+2}} \succeq \int_{0}^{(1-r) / 2} \frac{\rho d \rho}{(1-r|z|-\rho|z|)^{\alpha+\beta+1}} .
$$

Is not difficult to show that

$$
\int_{V^{n}} \frac{d m_{2 n}(\zeta)}{|1-\bar{\zeta} z|^{\alpha+\beta+2}} \succeq \frac{1}{(1-r|z|)^{\alpha+\beta}}
$$

Therefore

$$
\left\|\widetilde{P}_{\alpha}\left(g_{r}\right)\right\|_{B_{p}(\omega)} \succeq \frac{(1-r)^{\alpha p}}{\omega(1-r)} \int_{U^{n}} \frac{\omega(1-|z|)\left(1-|z|^{2}\right)^{\beta p}}{|1-r \bar{z}|^{(\alpha+\beta) p}\left(1-|z|^{2}\right)^{2}} d m_{2 n}(z)
$$

(we have used the inequality $1-r|z| \leq|1-r \bar{z}|$ ). If we assume that $\alpha_{j} p \leq \alpha_{\omega_{j}}-2$ for some $j$, then for the corresponding integral taking $\omega_{j}(t)=t^{\alpha_{\omega_{j}}}$ we get

$$
\int_{U} \frac{\left(1-\left|z_{j}\right|\right)^{\beta p-2}}{\left|1-r_{j} z_{j}\right|^{\left(\alpha_{j}+\beta\right) p}} \omega_{j}\left(1-\left|z_{j}\right|\right) d m_{2}\left(z_{j}\right) \sim \text { const }, \quad \text { if } \quad \alpha_{j} p<\alpha_{\omega_{j}}-2
$$

and

$$
\int_{U} \frac{\left(1-\left|z_{j}\right|\right)^{\beta p-2}}{\left|1-r_{j} z_{j}\right|^{\left(\alpha_{j}+\beta\right) p}} \omega_{j}\left(1-\left|z_{j}\right|\right) d m_{2}\left(z_{j}\right) \sim \log \frac{1}{1-r_{j}}, \quad \text { if } \quad \alpha_{j} p=\alpha_{\omega_{j}}-2
$$

Consequently,

$$
\frac{\left(1-r_{j}\right)^{\alpha_{j} p}}{\omega_{j}\left(1-r_{j}\right)} \rightarrow \infty, \quad \frac{\left(1-r_{j}\right)^{\alpha_{j} p}}{\omega_{j}\left(1-r_{j}\right)} \log \frac{1}{1-r_{j}} \rightarrow \infty
$$

if $r_{j} \rightarrow 1-0$.

Corollary 1. Let $L^{\infty}\left(U^{n}\right)$ be the set of all bounded measurable functions on $U^{n}$ and let $T_{h}(f)$ be the Toeplitz operator of the form $T_{h}(f)=P_{\alpha}(f h)$ 
with symbol $h \in L^{\infty}\left(U^{n}\right)$. If $\alpha_{j} p>\alpha_{\omega_{j}}-2(1 \leq j \leq n)$, then $T_{h}(f)$ is bounded on $B_{p}(\omega)$.

Furthermore, for $\alpha=\left(\alpha_{1}, \ldots, \alpha_{n}\right) \alpha_{j}>-1(1 \leq j \leq n)$ we define $R_{\alpha}(g)$ as follows

$$
R_{\alpha}(g)(z):=\frac{\prod_{j=1}^{n}\left(\alpha_{j}+1\right)}{\pi^{n}} \int_{U^{n}} \frac{g(\zeta)}{(1-\bar{\zeta} z)^{\alpha+2}} d m_{2 n}(\zeta)
$$

Theorem 5. Let $\alpha_{j} p>\alpha_{\omega_{j}}-2, \widetilde{\omega}_{j}(t)=\omega_{j}(t) t^{-\alpha_{j} p}(1 \leq j \leq n)$. Then the map $R_{\alpha}$ is bounded from $L_{p}(\widetilde{\omega})$ to $B_{p}(\omega)$. Moreover, if $p=1$ the map is surjective.

The proof of Theorem 5 is similar to the proof of Thoerem 4. We omit the details.

Remark 2. As in the case of $P_{\alpha}$, one can prove that the condition $\alpha_{j} p>\alpha_{\omega_{j}}-2(1 \leq j \leq n)$ in Theorem 5 is necessary, too( see Remark 1$)$.

We do not know whether the operators $P_{\alpha}$ and $R_{\alpha}$ in Theorem 5 and 6 are surjective for $p>1$, too.

We pass to the analysis of the right inverse map and consider the question: does there exist operators $R_{\alpha}^{-}\left(P_{\alpha}^{-}\right)$such that

$$
R_{\alpha}^{-}: B_{p}(\omega) \rightarrow L_{p}(\widetilde{\omega}) \quad \text { and } \quad P_{\alpha}^{-}: B_{p}(\omega) \rightarrow L_{p}(\omega)
$$

with

$$
R_{\alpha}\left(R_{\alpha}^{-}\right)(f)(z) \equiv f(z) \quad \text { and } \quad P_{\alpha}\left(P_{\alpha}^{-}\right)(f)(z) \equiv f(z), z \in U^{n}
$$

(where $\tilde{\omega}$ is the weight of Theorem 5 ). The answer turns out to be positive.

For $\beta$ and $\gamma$ let us consider the operator

$$
T_{\beta, \gamma} f(z)=\left(1-|z|^{2}\right)^{\beta+\gamma} \int_{U^{n}} \frac{\left(1-|\zeta|^{2}\right)^{\alpha}}{(1-\bar{\zeta} z)^{\beta+\alpha+2}} f(\zeta) d m_{2 n}(\zeta) .
$$

In the next theorem we discuss the boundedness of $T_{\beta, \gamma}$.

Theorem 6. Let $\alpha_{j}>\alpha_{\omega_{j}}-2, \beta_{j}>0(1 \leq j \leq n)$. Then

(1) $T_{\beta, \alpha}$ is bounded from $B_{p}(\omega)$ to $L_{p}(\widetilde{\omega})$ and $R_{\alpha}\left(T_{\beta, \alpha}\right) f(z) \equiv$ $f(z), z \in U^{n}$.

(2) $T_{\beta, 0}$ is bounded from $B_{p}(\omega)$ to $L_{p}(\omega)$ and $P_{\alpha}\left(T_{\beta, 0}\right) f(z) \equiv$ $f(z), z \in U^{n}$.

Proof. (1) Let $f \in B_{p}(\omega)$. First we show that $T_{\beta, \alpha}(f) \in L_{p}(\widetilde{\omega})$. By Theorem 5 there exist a function $g \in L_{1}(\widetilde{\omega})$ such that $R_{\alpha}(g)(z)=f(z), z \in$ $U^{n}$. Consequently 


$$
T_{\beta, \alpha}(f)(z)=\left(1-|z|^{2}\right)^{\beta+\alpha} \int_{U^{n}} \frac{g(\xi) d m_{2 n}(\xi)}{(1-\bar{\xi} z)^{\beta+\alpha+2}}
$$

Here we used $([15])$

$$
\int_{U^{n}} \frac{\left(1-|\xi|^{2}\right)^{\alpha}}{(1-\bar{\xi} w)^{\alpha+2}} \cdot \frac{d m_{2 n}(\xi)}{(1-\xi \bar{z})^{\alpha+\beta+2}}=\frac{1}{(1-w \bar{z})^{\alpha+\beta+2}}
$$

Therefore in the case $p=1$ we get, using Lemma 2,

$$
\begin{aligned}
\left\|T_{\beta, \alpha}(f)\right\|_{L_{1}(\widetilde{\omega})} & \preceq \int_{U^{n}}|g(\xi)| \int_{U^{n}} \frac{\omega(1-|z|)\left(1-|z|^{2}\right)^{\alpha+\beta} d m_{2 n}(z)}{|1-\bar{\xi} z|^{\alpha+\beta+2}\left(1-|z|^{2}\right)^{\alpha+2}} d m_{2 n}(\xi) \\
& \preceq \int_{U^{n}}|g(\xi)| \frac{\omega(1-|\xi|)}{\left(1-|\xi|^{2}\right)^{\alpha+2}} d m_{2 n}(\xi) \\
& =\|g\|_{L_{1}(\omega)} \preceq\|f\|_{B_{p}(\omega)} .
\end{aligned}
$$

Let $p>1$. Using Hölder's inequality and Theorem 1 we get

$$
\begin{aligned}
& \left\|T_{\beta, \alpha}(f)\right\|_{L_{p}(\widetilde{\omega})} \\
& \preceq \int_{U^{n}}|f(\xi)|^{p}\left(1-|\xi|^{2}\right)^{\alpha} \int_{U^{n}} \frac{\left(1-|z|^{2}\right)^{\beta-2}}{|1-\bar{\xi} z|^{\alpha+\beta+2}} \omega(1-|z|) d m_{2 n}(z) d m_{2 n}(\xi) \\
& \preceq \int_{U^{n}}|f(\xi)|^{p} \frac{\omega(1-|\xi|)\left(1-|\xi|^{2}\right)^{\beta-2}}{\left(1-|\xi|^{2}\right)^{\alpha+\beta}} d m_{2 n}(\xi)=\|f\|_{L_{p}(\omega)} \preceq\|f\|_{B_{p}(\omega)}<\infty
\end{aligned}
$$

A calculation of the corresponding integrals shows, that

$$
\frac{\prod_{j=1}^{n}\left(\alpha_{j}+1\right)}{\pi^{n}} \int_{U^{n}}\left(1-|\xi|^{2}\right)^{\alpha} f(\xi) \int_{U^{n}} \frac{\left(1-|\zeta|^{2}\right)^{\alpha+\beta}}{(1-\bar{\xi} \zeta)^{\alpha+\beta+2}} \frac{d m_{2 n}(\zeta) d m_{2 n}(\xi)}{(1-\bar{\zeta} z)^{\alpha+2}}=f(z) .
$$

Therefore $R_{\alpha}\left(T_{\beta, \alpha}\right)(f)(z)=f(z), z \in U^{n}$. We have also used Theorem 2.1 of [5].

(2) To prove that $T_{\beta, 0}: B_{p}(\omega) \rightarrow L_{p}(\omega)$ we use Theorem 4 and Hölder's inequality. As in the case of $T_{\beta, \alpha}$ we have

$$
\left\|T_{\beta, 0}\right\|_{L_{p}(\omega)} \preceq\|g\|_{L_{p}(\omega)} \preceq\|f\|_{B_{p}(\omega)}
$$

and a calculation shows that $P_{\alpha}\left(T_{\beta, 0}\right)(f)(z) \equiv f(z), z \in U^{n}$.

\section{References}

[1] J. Arazy and S.Fisher, The uniqueness of the Dirichlet spaces among Möbius invariant Hilbert spaces, Illinois J. Math. 29 (1985), 449-462. 
[2] J. Arazy, S. Fisher and J. Peetre, Möbius invariant function spaces, J. Reine Angew. Math., 363 (1985), 110-145.

[3] O. Blasco, Multipliers on weighted Besov spaces of analytic functions, Contemporary Mathematics, 144 (1993), 23-33.

[4] M.M. Djrbashian, On the representation problem of analytic functions, (in Russian), Soobsh. Inst. Matem. Mekh. Akad. Nauk Arm SSR (1948), 3-40.

[5] A. Harutyunyan, Description of some weighted spaces of holomorphic functions in terms of fractional derivatives, Complex Variables and Elliptic Equations, 51 (2006), 1103-1113.

[6] A. Harutyunyan, Weighted Bloch space in the polydisk, J. Funct. Spaces Appl., 5 (2007), 213-230.

[7] A. Harutyunyan and W. Lusky, Duals of holomorphic Besov spaces on the polydisk and diagonal mappings, in preparation.

[8] H. Hedenmalm, B. Korenflum and K. Zhu, Theory of Bergman Spaces, Springer, New York, 2000.

[9] A.N. Karapetyants and F.D.Kodzoeva, Analytic weighed Besov spaces on the unit disk, Proc. A. Razmadze Math.Inst., 139 (2005), 125-127.

[10] W. Rudin, Theory of Functions in the Unit Ball of $C^{n}$, Springer, Berlin, 1980.

[11] E. Seneta, Regularly Varying Functions, Lect. Notes Math., 508, Springer, Berlin, 1976.

[12] F. Shamoyan, Diagonal mappings and questions of representation of anisotropic spaces in polydisk (in Russian), Sibisk. Mat. Zh. 3 (1990), 197-215, translation in Siberian Math. J. 31 (1990), 350-365.

[13] K. Stroethoff, Besov type characterisations for the Bloch space, Bull. Aust. Math. Soc., 39 (1989), 405-420.

[14] B. Yorikke, A multidimensional analogy of Privalov theorem, Math. Nachr. 107 (1982), 221-233.

[15] K. Zhu, Operator Theory in Function Spaces, Marcel Dekker, New York, 1990.

Fac. for Inf. and Appl. Math., University of Yerevan

Alek Manukian 1, Yerevan 25, Armenia

(E-mail : anahit@ysu.am)

Inst. for Math., University of Paderborn

Warburger Str. 100, D-33098 Paderborn, Germany

(E-mail : lusky@uni-paderborn.de)

(Received : March 2008) 


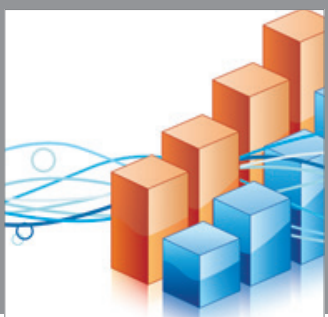

Advances in

Operations Research

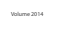

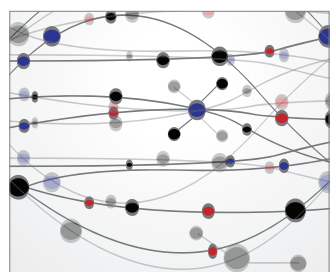

\section{The Scientific} World Journal
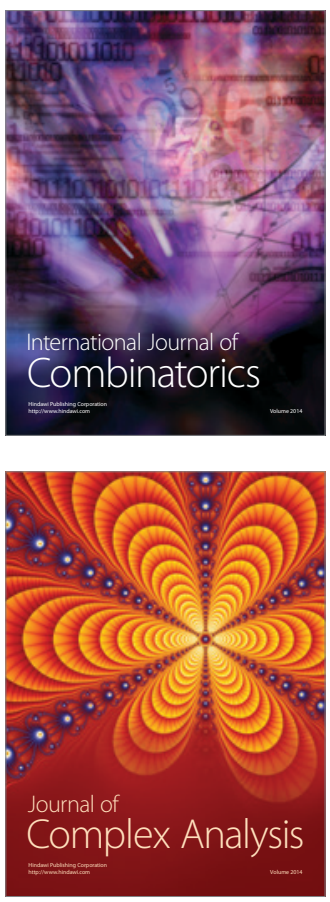

International Journal of

Mathematics and

Mathematical

Sciences
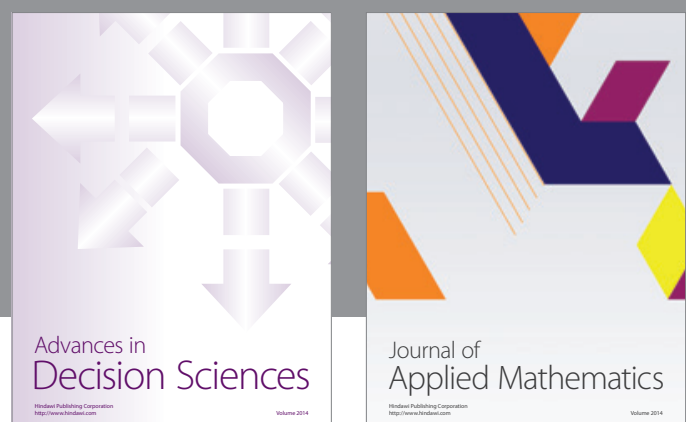

Journal of

Applied Mathematics
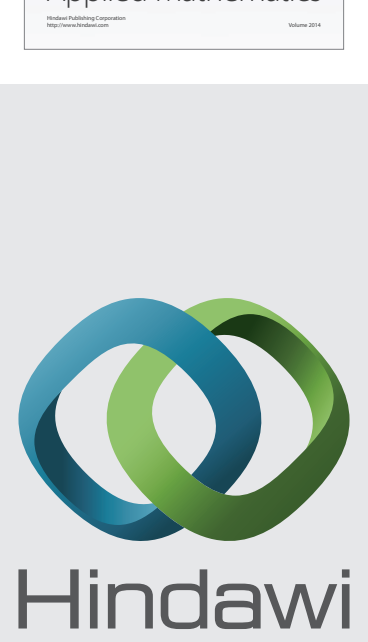

Submit your manuscripts at http://www.hindawi.com
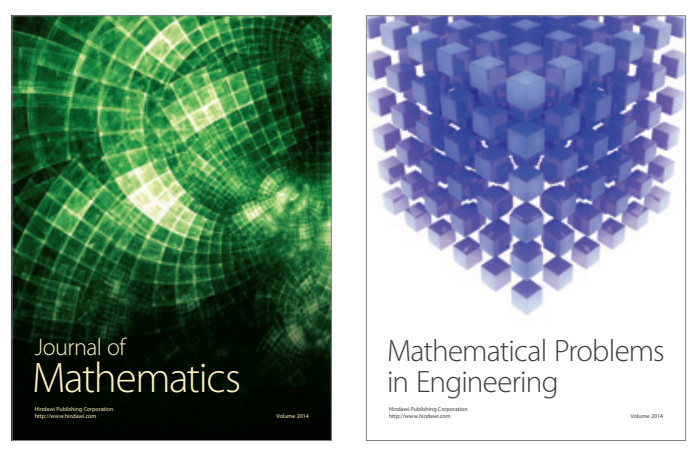

Mathematical Problems in Engineering
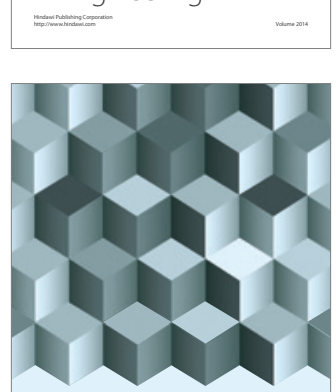

Journal of

Function Spaces
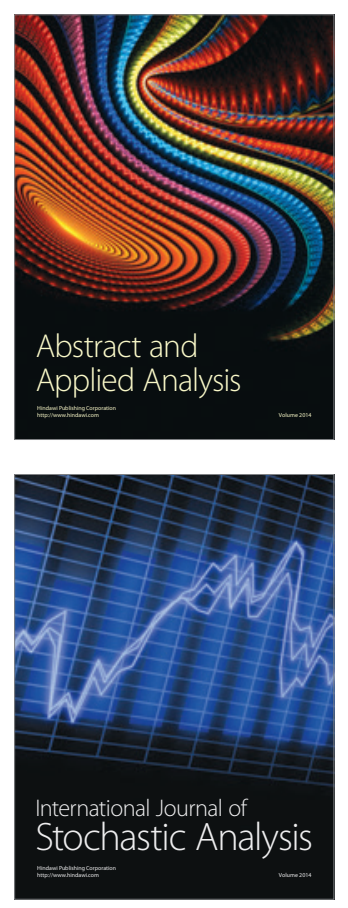

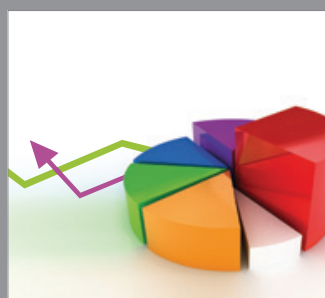

ournal of

Probability and Statistics

Promensencen
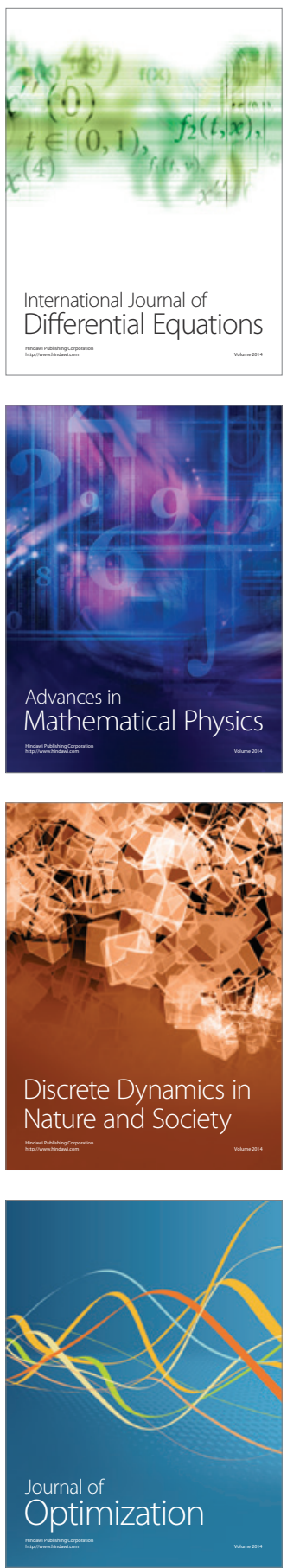